\title{
Análisis de un sistema de información geográfica para la interpretación de cartas de campo magnético y geológico del semidesierto de Zacatecas
}

\author{
Interpretation of magnetic field and geological charts of the \\ semidesert of Zacatecas
}

Héctor Miguel Casas Flores'*

Casas Flores, H. M. Análisis de un sistema de información geográfica para la interpretación de cartas de campo magnético y geológico del semidesierto de Zacatecas. Investigación y Ciencia de la Universidad Autónoma de Aguascalientes. Número 70: 5-12, enero-abril 2017.

\section{RESUMEN}

El semidesierto de Zacatecas ha sido un gran impulsor del desarrollo económico que se ha sostenido a nivel industrial debido a la cantidad, calidad y cotización de minerales que se encuentran dentro de las 13 imponentes formaciones litológicas del estado. La región de estudio abarca aproximadamente 3,744 $\mathrm{km}^{2}$ y se caracteriza por tener rocas de composición basáltica, riolítica, rocas intrusivas como granodioritas, monzonitas de la Era Cenozoica (Terciaria) y también rocas metamórficas caracterizadas por el tipo de metamorfismo de contacto de grado medio a bajo.

El análisis de multivariables fue utilizado para construir diagramas jerárquicos (dendrogramas) y mapas de similitudes. Los principales puntos magnéticos de alta susceptibilidad magnética lo obtienen las formaciones de la Era Cuaternaria, Terciaria, Cretácica Temprana y Jurásica Tardía, lo que implica que sobre estas capas de roca y en

Palabras clave: formación litológica, metamorfismo de contacto, análisis multivariado, diagramas jerárquicos, susceptibilidad magnética, estructuras minerales.

Keywords: lithological formation, multivariate analysis, hierarchical diagrams, magnetic susceptibility, mineral structures.

Recibido: 25 de junio de 2015, aceptado: 21 de marzo de 2016

Maestría en Ingeniería Aplicada con Orientación en Recursos Hidráulicos, Universidad Autónoma de Zacatecas, México.

* Autor para correspondencia: hcmcasas@gmail.com algunas estructuras minerales profundas se obtenga el mayor contenido de minerales ferromagnéticos.

\section{ABSTRACT}

The semidesert region of Zacatecas has been a major driver of economic development sustained at an industrial level by the quantity, quality and price of the minerals found in 13 stunning lithological formations in this state. The study area of approximately 3,744 $\mathrm{km}^{2}$ is characterized by rocks of basaltic composition, rhyolite, intrusive rocks such as granodiorite, monzonite of the terciary period at the beginning of the Cenozoic Era, and also low to medium grade metamorphic rocks also characterized by the type of contact metamorphism of low average degree.

Multivariate analysis was used to build hierarchical diagrams (dendrograms) and maps of similarities. The main spots of high magnetic susceptibility were obtained to be in formations from the quaternary, terciary, early Creataceous and late Jurassic periods, which implies that the highest content of ferromagnetic minerals is to be found in this layers of rock and in certain deep mineral structures.

\section{INTRODUCCIÓN}

La región del semidesierto de Zacatecas tiene importancia a nivel internacional, debido a que allí se localizan las principales formaciones geológicas que aumentan la explotación y producción futuras de minerales de fierro y magnesio. El oro se encuentra 
IIVESTIGACIÓn Y CIERCIA DE LA UNIVERSIDAD AUTÓNOMA DE AGUASCALIERTES asociado a la mineralización de especularita, hematita, calcopirita y pirita. Las amplias variedades de rocas en la región, desde minerales metálicos hasta los no metálicos, permiten al geocientífico aportar e indagar el área de estudio con métodos actuales.

Con el resultado del método aplicado, que fue un análisis multivariable, se demuestra adecuadamente que hay importantes concentraciones de minerales en diferentes partes de las localidades del semidesierto zacatecano, y que debido a los emplazamientos, yacimientos, estructuras y formaciones que varían de dimensión, se pueden obtener interesantes valores de hematita, magnetita, cobre y oro, los cuales son sustanciales y necesarios para la industria en general.

El área de estudio se encuentra en la porción semiárida que comprende el estado de Zacatecas y se ubica entre las coordenadas $24^{\circ} 45^{\prime}, 24^{\circ} 15^{\prime}$ de latitud norte a $101^{\circ} 20^{\prime}, 101^{\circ} 40^{\prime}$ de longitud oeste. Ha sido afectada por eventos geológicos durante la Era Mesozoica que consistieron en cambios tectónicos consecuentemente acompañados por amplios eventos volcánicos producidos en tiempos laramídicos (Damon et al., 1981).

El basamento consiste en rocas metamórficas y sedimentarias proterozoicas, producto de los bloques de Oaxaquia, como indican Centeno García et al. (2008). Actualmente, el área de estudio forma parte de una zona que se encuentra en constante crecimiento de actividades de exploración y explotación de minerales dada su importancia económica, como el oro, plata, cobre y zinc. Existen varios estudios geológicos y geofísicos realizados en esta región por empresas, en los que se percataron de ciertas anomalías magnéticas y permitieron delimitar algunas estructuras minerales como es el caso de Chile Colorado, Brecha Azul, Brecha, entre otros documentados por el Servicio Geológico Mexicano (SGM, 2004a y b).

El objetivo del presente artículo fue lograr un agrupamiento de las unidades de rocas encontradas en el área de estudio para emplearlo en cartografía geológica y otras áreas del conocimiento. Esta técnica puede ser utilizada para la relación de litologías, reconocimiento potencial de contenido de altos puntos magnéticos y observación de anomalías magnéticas. Otra técnica nombrada en este trabajo es el llamado gradiente vertical, sinónimo de la definición de derivada vertical por Milligan y Gunn (1997), físicamente equivalente a la medición de campo magnético simultáneamente en dos puntos verticalmente, uno encima del otro, substrayendo los datos y dividiendo los resultados entre la separación vertical espacial de los puntos de medición.

De lo nombrado anteriormente, se dará un entendimiento del panorama magnético que permita a su vez promover beneficios e información adicional que sea trascendente a la hora de aprovechar recursos naturales. Los resultados sugieren que los valores de los principales puntos magnéticos de alta susceptibilidad magnética que son las localidades El Sol y La Luna, La Felicidad y La Escondida, se obtienen de las formaciones que datan del Cretácico Temprano y Jurásico Tardío y las formaciones litológicas son parte de una fuente autóctona, como las rocas cuaternarias y terciarias.

MATERIALES Y MÉTODOS

El análisis consistió en varias fases. Las más importantes fueron dos: en la primera fueron revisadas las variables del campo magnético total (CMT) con respecto a las formaciones litológicas y la segunda fue la mención de la técnica del gradiente vertical, de la reducción al polo con respecto a las formaciones litológicas. Para el análisis de las estructuras litológicas que se encuentran dentro de la región, se utilizó un método multivariables que consistió en un análisis de conglomerados (cluster en inglés) y un agrupamiento jerárquico de datos (AJD), definido por Facchinelli et al. (2001). Algunos métodos y estudios similares han sido integrados en bases de datos sobre el noreste de México, que según Aiken et al. (1997), en colaboración con el Instituto de Geografía de la UNAM en 1990, contribuyeron a la generación del modelamiento por computadora e interpretación de la corteza sísmica con métodos gravimétricos que para las formaciones geológicas que construyen la corteza terrestre hasta cierta profundidad es determinada por el alcance de este método. Las rocas de mayor densidad aumentan la aceleración y se realizan mediciones; es decir, se miden las atracciones laterales de la fuerza gravitatoria de un lugar al otro y posteriormente se intentan relacionar con las características geométricas del cuerpo, así como de sus contrastes de densidades. Algunas de las revisiones del modelamiento por computadora 
para propósitos de interpretación fueron realizadas a finales de la década de los setenta del siglo XX por el ingeniero hindú Bhattacharyya (1978).

Para cubrir los objetivos propuestos en este trabajo se consultaron las cartas geológicas mineras y magnéticas del SGM, así como diversos trabajos que contienen metadatos y reportes sobre temas de carácter geológico y geofísico-magnético. Se hizo un procesamiento de 11 cartas de escala 1:50,000, de las cuales, cuatro fueron cartas geológicas-mineras, cuatro geofísicas de carácter magnético y tres de derivada vertical, debido a que la carta magnética del primer gradiente vertical correspondiente a Concepción del Oro en 2013 no había sido realizada porque para producirlas se requiere equipo especializado como magnetómetros y programas de cómputo más sofisticados, como el procesamiento de datos en un sistema informático llamado Oasis. Hay cuatro localidades del municipio de Mazapíl, Concepción del Oro G14-C62, con curvas 40 nT, Cedros G14-C61 con curvas cada $20 \mathrm{nT}$ y $4 \mathrm{pT} / \mathrm{m}$, El Cardito G14-C71 con curvas cada $10 \mathrm{nT}$ y $2 \mathrm{pT} / \mathrm{m}$ y Tanquecillos G14-C72 con curvas de 20nT y $10 \mathrm{pT} / \mathrm{m}$, las que destacamos por su importancia geológica. Para la realización de este artículo no se hizo ningún trabajo de campo, básicamente consistió en la creación y procesamiento de datos vectoriales, cuya información se adquirió de las cartas geológicas antes mencionadas.

Se capturaron capas de información geográfica del campo magnético total (CTM), gradiente vertical y litológica en formato vectorial. La sobreposición de información con la mezcla de sus atributos, mediante capas de entrada, se complementa hasta que se consiga otra capa deseada, esto fue explicado por Davis (2001). Las operaciones permiten que la información de una capa sea superpuesta sobre la información de otra donde, dependiendo de los objetos, se pueden obtener diferente información de salida. En la clasificación de los datos se aplicó un método multivariado para agrupar instancias (variables) que permite el reconocimiento de patrones.

El método utilizado consistió en un análisis de agrupación jerárquica del tipo de proceso por conglomerados acumulativos (cluster analysis) propuesto por Jain y Dubes (1988) y para la visualización de la información se utilizaron gráficos jerárquicos que en inglés son conocidos como dendograms (dendogramas) y constituyen la representación que mejor ayuda a interpretar un análisis de conglomerados. La técnica de agrupación jerárquica es apropiada para mostrar las relaciones de las propiedades físicas de las rocas. Este análisis de grupos jerárquicos es una técnica exploratoria que revela agrupaciones naturales y representa, a su vez, las relaciones con las formaciones de las rocas. El paquete informático de visualización geográfica fue de gran utilidad; correspondientemente fue seleccionado conforme a su capacidad de visualizar las relaciones espaciales entre el ambiente de datos y las características de la superficie de la región.

\section{RESULTADOS}

\section{Sobreposición de capas del campo total magnético contra las rocas}

El análisis tuvo varias fases, las más importantes fueron dos: en la primera se analizaron variables del campo magnético total (CMT) con respecto a las formaciones litológicas y en la segunda fue la mención de la técnica del gradiente vertical, de la reducción al polo con respecto a las formaciones litológicas, según Milligan y Gun (1997). Se aclara que los valores obtenidos en tablas es la sumatoria de toda la geometría de capas geográficas creadas en las cartas litológicas.

\section{Campo magnético total contra formación litológica}

La formación Cupido ocupa mayor extensión superficial en el área de estudio, tiene 24,102.08 ha y es seguida por la formación Cuesta del Cura, la cual tiene 12,168.25 ha. Además, se encuentran tres formaciones litológicas que contribuyen a los valores de alto gradiente magnético y altos valores de intensidad magnético de 2,100 nT, mismas que pertenecen a las formaciones Cupido, Taraises y La Caja, sus valores de desviación estándar están sobre $747.52 \mathrm{nT}$ y $770.61 \mathrm{nT}$, por lo que sus datos son dispersos, lo que provoca una variación grande con respecto al bajo gradiente del campo magnético total. Consecuentemente, debajo de estos valores se encuentra la formación Zuloaga, con valor de 2,085 nT y promedio de 791 nT. Esto evidencia cuerpos ocultos mineralizados en estas tres unidades litológicas, lo que causa la aparición de anomalías magnéticas en la región y, en consecuencia, entre sus capas se asimila un papel preponderante en la preservación de los depósitos minerales, lo que a su vez causa que se encuentren algunos halos de metamorfismo originados por el contacto de las 
IIVESTIGACIÓn Y CIERCIA DE LA UNIVERSIDAD AUTÓNOMA DE RGUASCALIERTES secuencias sedimentarias con el cuerpo intrusivo. Ello produce gran porcentaje de mineralización compuesta por óxidos y sulfuros de fierro encima de la otra capa secuencial de la formación Zuloaga, esta puede ser la razón por la que en la Figura 1 se exponen altos valores de susceptibilidad para rocas sedimentarias marinas. En general, el contenido de magnetita es extremadamente variable y la susceptibilidad de las rocas puede ser considerable en el solapamiento entre diferentes litologías. Las rocas metamórficas también son variables en su carácter magnético, si la presión de oxígeno parcial es relativamente baja, la magnetita es reabsorbida, el fierro y oxígeno son incorporados dentro de otra fase mineral como el grado de metamorfismo incrementa (Kearey et al., 2002).

La desviación estándar de 5.91 a 23.50 nT y promedios de $-40,-49$ y -11 nT de las formaciones Nazas, La Joya y Taray exhiben un bajo gradiente magnético. Esto implica que los datos de la desviación estándar no son muy altos, están cerca de los valores promedio, la formación Taray es el basamento y es de gradiente menor, también tiene la característica de ser no magnético, decrece la cantidad de minerales ferromagnéticos.

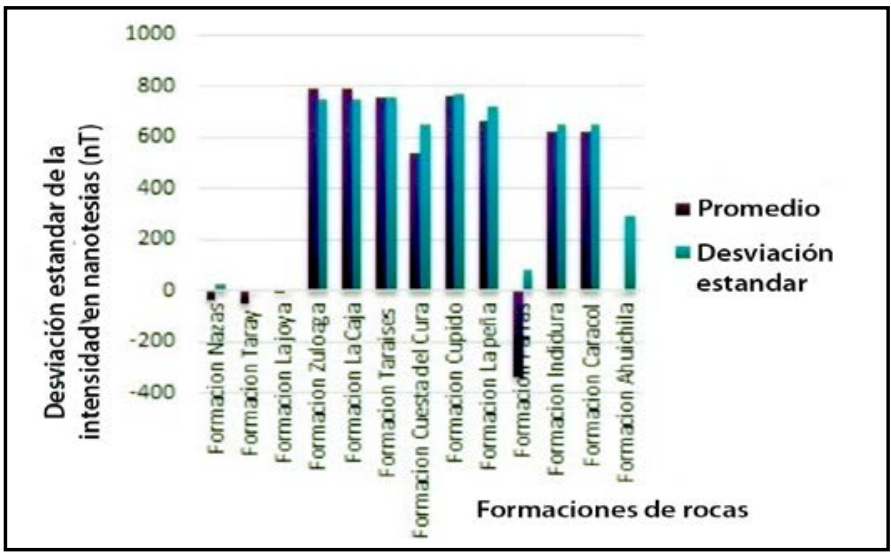

Figura 1. Gráfica de los valores de las diferentes formaciones de rocas y la distribución con los índices más altos y más bajos. Elaboración propia con información obtenida de las cartas consultadas en el SGM (1997, 1998, 2004, 2005).

Para la clasificación de los elementos en conglomerados se utilizaron diagramas jerárquicos que partiendo de un solo grupo van separados de los demás elementos y los cuales, cada vez se van haciendo más pequeños. A partir de estas unidades iniciales se van formando grupos de forma ascendente; las líneas superior e inferior con valores

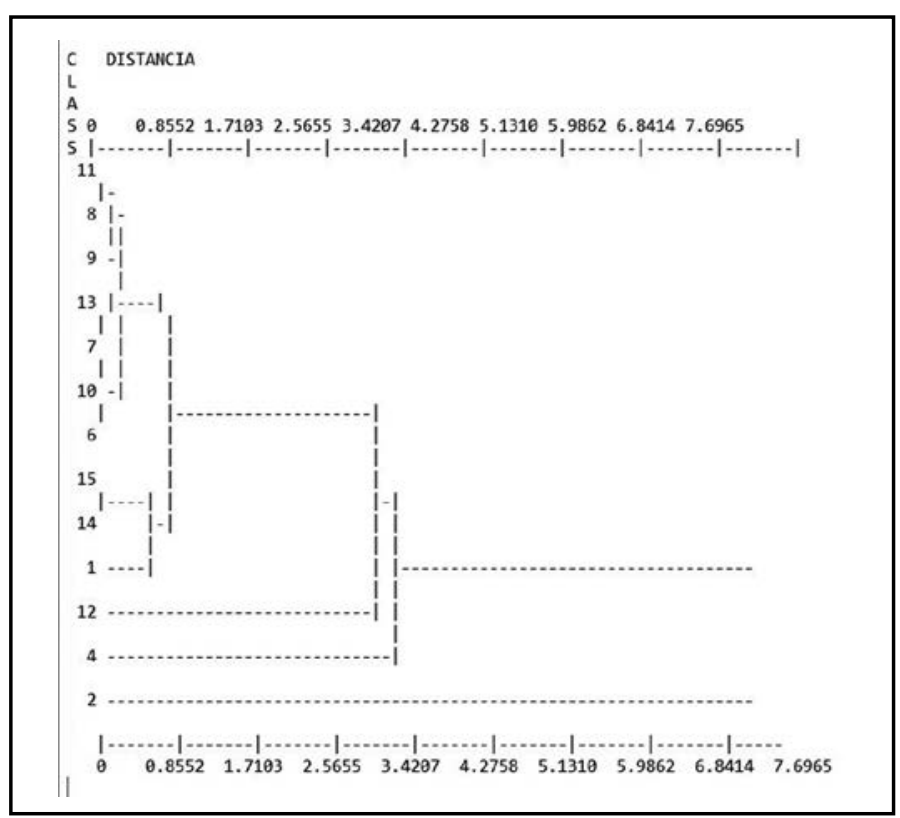

Figura 2. Análisis del diagrama jerárquico. Elaboración propia con información obtenida de las cartas consultadas del SGM $(1997,1998,2004,2005,2009)$.

ascendentes indican que cada elemento entre más se aleje de cero disminuye la similitud con respecto a sus componentes minerales; las observaciones más distantes son 4 y 12 las únicas mayores distancias y la 2, que no tiene grupo. La observación más cercana de elementos con menor distancia (más cercanas a cero) es 11, 8, 9, 13, 7, 10, 6, 15, 14. En el dendograma de las variables campo magnético total (CMT) y formaciones geológicas, las formaciones La Peña (11) y Taraises (8) forman un grupo en el primer nivel; las formaciones Indidura (13), La Caja (7), Cupido (10) y Zuloaga (6) forman el segundo grupo en el primer nivel. Las formaciones Ahuichila (15) y Caracol (14) se encuentran formando el tercer agrupamiento.

El primer grupo de rocas representado por las formaciones La Peña, Taraises, Cuesta del Cura, Indidura, La Caja, Cupido y Zuloaga, está asociado a los cambios progresivos del magma intermedio que se solidifica como cuerpo intrusivo dentro de estas capas de roca encajonante; el magma enriquecido interactúa con las diferentes unidades litológicas, que es la posible razón de anomalías fuertes y positivas magnéticas.

Las formaciones Caracol y Ahuichila están controladas por una clara asociación, sus contactos superiores se encuentran adyacentes, caracterizados por basaltos y sedimentos lacustres a consecuencia 


\section{IIVESTIGAGIÓn Y CUERCIA DE LA UחIVERSIDAD AUTÓNOMA DE AGUASCALIERTES}

de efectos tectónicos; son formaciones afectadas por intensos plegamientos y probablemente comparten componentes minerales en estos depósitos continentales.

\section{Gradiente vertical contra formación litológica}

Si se toma en cuenta la sobreposición de capas de atributos de la derivada vertical, la combinación de los valores de las formaciones que están distribuidas en la región y los datos arrojados del campo magnético total, se obtiene que las formaciones Cuesta del Cura, La Peña y Cupido tienen los valores más altos de $282 \mathrm{pT} / \mathrm{m}, 280 \mathrm{pT} / \mathrm{m}$ y $279 \mathrm{pT} / \mathrm{m}$, y sus valores mínimos, de -204 pT/m,-200 pT/m y -200 pT/m, implican que Cuesta del Cura es una de las unidades litológicas que conforma núcleos significativos de áreas mineralizantes granodioríticas y que por alguno de los diversos efectos como la presión parcial de alto oxígeno pudieron haber producido minerales accesorios como ilmenita, piroxenos, esfena y biotita, alanita, pirita Clark (1997); en la Figura 3 se reflejan sus diferentes capas y agrupaciones. Los valores máximo y mínimo se muestran en negritas en la Tabla 1.

Las observaciones que se percibieron en la formación Parras, composición de lutitas fisibles, consisten en que sus dominios magnéticos carecen de sus valores de intensidad, por lo que las capas litológicas no son geológicamente magnéticas debido a que las partículas de roca en su mayoría están estructuradas por minerales detríticos como arcillas de $0.0039 \mathrm{~mm}$, los más finos en las rocas. Es improbable que entre sus minerales accesorios se encuentren minerales ferromagnéticos debido a su probable alteración del contenido de carbonato de sodio.

Como en el dendrograma anterior, las líneas superior e inferior con valores ascendentes indican que cada elemento entre más se aleje de cero disminuye la similitud con respecto a sus componentes minerales. Las observaciones más distantes son los grupos de formación Indidura (13), que no tiene grupo, skarn, hornfels (2), mármol (1), formación Caracol (14) y filita-arenisca (4). Las observaciones más cercanas, que son elementos con menor distancia de cero corresponden a 11, 8, 9, 15, 6, 7. En el dendrograma de las variables de gradiente vertical y formaciones geológicas se observan cuatro grupos, de los más significativos son 2: la formación La Peña (11), Taraises (8), forman el primer grupo correspondiente al primer nivel. De las formaciones Ahuichila (15) y Zuloaga (6) se forma el segundo agrupamiento, las rocas skarn, hornfels (2) y mármol (1) forman el tercer grupo y la formación Caracol (14) y filita-arenisca (4) forman el cuarto.

Tabla 1. Conjunto de valores de las capas de rocas de formaciones geológicas con relación al gradiente de reducción que muestra su índice bajo como el más alto

\begin{tabular}{|l|r|c|c|c|c|}
\hline \multicolumn{1}{|c|}{ Formación } & $\begin{array}{c}\text { Superficie } \\
\text { ha }\end{array}$ & $\begin{array}{c}\text { Mínimo en } \\
\mathbf{p T} / \mathbf{m}\end{array}$ & $\begin{array}{c}\text { Máximo en } \\
\mathbf{p T} / \mathbf{m}\end{array}$ & $\begin{array}{c}\text { Promedio } \\
\text { en } \mathbf{~ p T / m}\end{array}$ & $\begin{array}{c}\text { Desviación } \\
\text { estándar } \mathbf{p T / m}\end{array}$ \\
\hline Formación Nazas & $1,578.37$ & -14 & 21 & 4 & 10.53 \\
\hline Formación Taray & 56.2 & -1 & 3 & 1 & 1.58 \\
\hline Formación La Joya & 25.22 & -14 & -3 & -9 & 3.60 \\
\hline Formación Zuloaga & 10,053 & -210 & 269 & -9 & 3.60 \\
\hline Formación La Caja & 2105 & -210 & 199 & -7 & 117.51 \\
\hline Formación Taraises & $3,448.05$ & -210 & 227 & 5 & 125.19 \\
\hline Formación Cuesta del Cura & $\mathbf{1 8 , 4 8 8 . 2 1}$ & -200 & $\mathbf{2 8 2}$ & 41 & 139.57 \\
\hline Formación Cupido & $16,985.51$ & -204 & 279 & 38 & 139.86 \\
\hline Formación La Peña & 2,809 & -200 & 280 & 39 & 138.24 \\
\hline Formación Parras & $\mathbf{0}$ & $\mathbf{0}$ & $\mathbf{0}$ & $\mathbf{0}$ & $\mathbf{0}$ \\
\hline Formación Indidura & $1,042.62$ & -124 & 59 & -33 & 53.26 \\
\hline Formación Caracol & $2,805.44$ & -120 & 69 & -35 & 50.74 \\
\hline Formación Ahuichila & 11.16 & -20 & 51 & 16 & 20.92 \\
\hline
\end{tabular}

Elaboración propia con información obtenida de las cartas consultadas en el SGM (1997, 2004, 2005, 2009, 2010). 
IIVESTIGACIÓn Y CIERCIA DE LA UחIVERSIDAD AUTÓกOMA DE RGUASCALIERTES

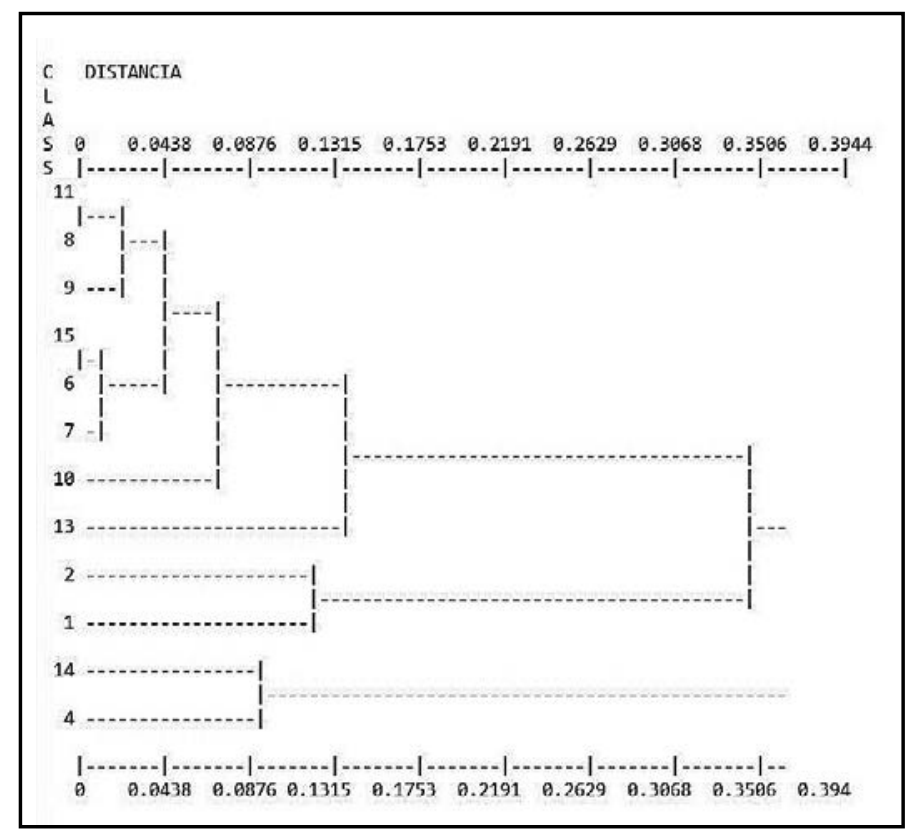

Figura 3. Análisis del diagrama Jerárquico de gradiente vertical magnético y formaciones geológicas. Elaboración propia con información obtenida de las cartas consultadas del SGM (1997. 2004, 2005, 2009, 2010).

El resultado estadístico del primer grupo fue menos concluyente, mientras que el agrupamiento 2 confirma la explicación mineralógenico-magmática, quedan algunas dudas acerca de los grupos 3 y 4 . Las posibles explicaciones son las siguientes:

- Las formaciones La Peña y Taraises, en el grupo 1, al igual que el grupo 2 de las formaciones Ahuichila y Zuloaga, están asociadas con otro factor mineralogénico; por ejemplo la presencia de rocas con sulfuros del mismo cuerpo intrusivo que los atraviesa.

- En las rocas skarn, hornfels y mármol están controladas por el proceso metamórfico regional y si se analiza la variabilidad somera del skarn es principalmente controlada por algunas anomalías magnéticas fuertes $y$ positivas alrededor de los intrusivos riolíticos y granodioríticos en las localidades de Noche Buena, Concepción del Oro y Terminal de Providencia.

\section{DISCUSIÓN}

Los resultados derivados del presente estudio muestran los puntos interesantes de susceptibilidad magnética de uno de los semidesiertos más importantes de México y Latinoamérica. Estos

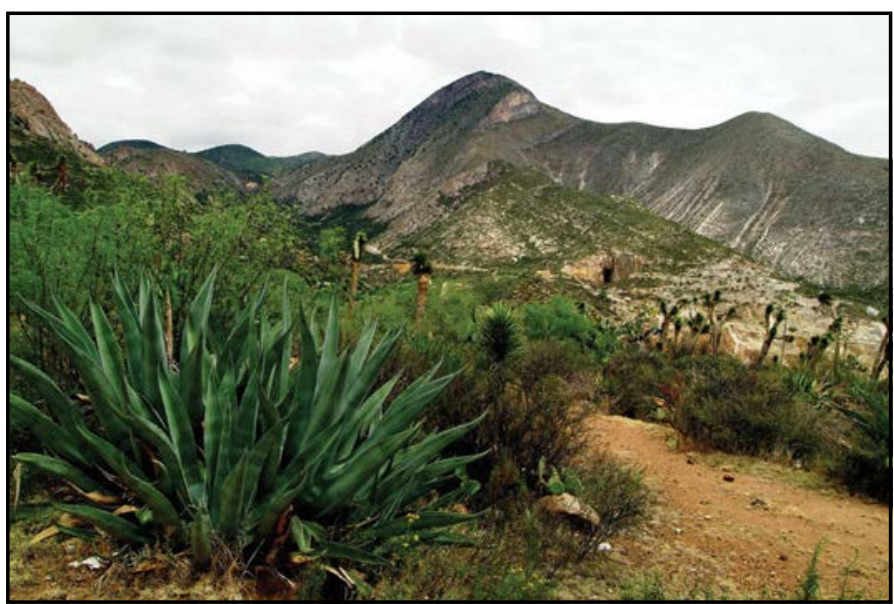

Figura 4. La sierra en Concepción del Oro, Zacatecas, México. Fotografía tomada por Antonio Ambriz Avendaño.

valores de susceptibilidad magnética se encuentran apegados a los datos expuestos obtenidos de las cartas litológicas y magnéticas de Cedros, Concepción del Oro, El Cardito y Tanquecillos expuestos en el SGM (1997, 1998, 2004, 2005, 2009, 2010) y representan una seria comprensibilidad debido a las implicaciones que el sector minero tiene sobre los recursos naturales.

Si se revisan los estudios y la aportación de datos de las cartas geológicas del SGM (2004), el valor alto magnético coincide en el área de Concepción del Oro, el área Noche Buena y Santa Rosa; tres de las más productivas de México. Se explica debido al mineral granodiorítico asociado al intrusivo que genera un mineral llamado skarn, en donde las principales estructuras formadas son chimeneas de dimensiones de hasta $500 \mathrm{~m}^{2}$, en donde se destacan las localidades El Capulín, Sol y Luna, La Escondida, La Laja, La felicidad, entre otros. Dentro de las rocas Mesozoicas, las formaciones Taraises, La Caja, Cupido y La Peña, como se explicó anteriormente, son formaciones donde sus estructuras minerales se encuentran profundas y ocultas.

Las diferencias de estos valores con respecto a los estudios de otras zonas del país no son similares. De acuerdo con un estudio de López Loera y Tristán González (2013), en el altiplano central de la parte centro oriental en el norte de la sierra San Miguelito, los valores son bajos con respecto a sus anomalías magnéticas de bloques para patrones de fallamientos normales, asimismo, para las anomalías de características negativas. 
Mediante uno de los métodos más elegantes: la agrupación jerárquica del tipo de proceso por conglomerados acumulativos (cluster analysis) se puede aceptar el cumplimiento de agrupación para cada una de las formaciones litológicas, a su vez expresaría que estos patrones de agrupamiento operan no solo para formaciones litológicas y capas de roca, sino también para una buena parte de fenómenos aglomerativos, susceptibles de ser clasificados jerárquicamente como valores anómalos geoquímicos, entre otros. Es por ello que se recomienda reportar los resultados en estas representaciones gráficas para enriquecer el análisis con este modelo estadístico.

Cabe destacar que es escasa la literatura que aborda las características magnéticas en el estado de Zacatecas se exhiben más bien una serie de reportes de diferentes localidades y municipalidades en la que el autor de este trabajo cree que esta versión actual es una contribución interesante que resulta de una apertura de proceso de datos. Finalmente, el área del semidesierto y el país cuenta con varias contingencias de estudio, que actualmente ha colocado a México como líder de la producción, importación y exportación de oro.

CONCLUSIONES

Se utilizaron 11 mapas magnéticos y geológicos de la red SGM para conocer los agrupamientos jerárquicos, de los cuales esta investigación se benefició para el análisis de la región del semidesierto de Zacatecas, en especial algunas localidades del municipio de Mazapíl. Los resultados principales se obtuvieron de la información de los mapas del campo magnético total para el análisis multivariado de las diferentes capas geológicas y por medio de agrupación e interpretación se identificaron varias relaciones.

Las rocas de pórfido riolítico y tipo skarn y hornfels tienen similitudes en sus valores de intensidad del campo magnético total. Debido a su parentesco en remanencia y susceptibilidad magnética, ambos grupos de rocas están asociados con los procesos magmáticos. Las formaciones La Peña, Taraises, Cuesta del Cura, Indidura, Caja, Cupido y Zuloaga constituyen grupos y sus capas de rocas tienen altos valores de magnetización. La magnetización de estas rocas sedimentarias marinas es producida por el magma enriquecido que interactúa con las diferentes unidades litológicas, que es la posible razón de anomalías magnéticas fuertes y positivas; además, estos datos significan que debido a su contenido magnético semejante, las capas se encuentran solapadas posiblemente con intrusivos de la región asociados a un proceso de acreción en la porción de la mesa central. Este tipo de fenómeno sucede cuando cierta cantidad de sedimentos son empujados contra los continentes y sufren metamorfismo de alta presión, esto se puede asociar con la deformación del Cretácico Tardío y con la sucesión de cabalgamientos y depósitos originados por la Orogenia Laramide.

Referente al gradiente magnéticoy formaciones litológicas, la variabilidad de las formaciones La Peña y Taraises, ambas asociadas, es controlada por rocas padre de rocas magmáticas. El paquete informático SIG muestra datos de relaciones espaciales entre anomalías positivas y negativas en relación con minerales ferromagnéticos.

Por último, el agrupamiento de la edad del campo magnético total es similar al grupo de los datos de la derivada de reducción en el sentido en que fueron agrupados en relación con sus diferentes valores magnéticos, son más suaves, por lo que el gradiente es menos abstracto. Los valores obtenidos muestran que los principales puntos magnéticos de alta susceptibilidad (área de Noche Buena, Concepción del Oro y Santa Rosa) pertenecen a las formaciones de la Era Cretácica Temprana y Jurásica Tardía, que probablemente resultaron de una fuente autóctona, al igual que las rocas de la Era Cuaternaria y Terciaria.

\section{Futuro de la investigación}

Los sistemas de información geográfica son usados por miles de geocientíficos, técnicos y continúan expandiéndose. Esto ha provocado que el uso de esta tecnología haya sido asimilada por universidades, gobierno, industria e instituciones en las que actualmente el SIG (paquete informático) provee de acceso y conexión a internet para poder acceder a nuevos subconjuntos de datos y opciones de operatividad vía satélite, en los que varias disciplinas en conjunto tratan de entender los patrones, procesos y algunas consecuencias que tienen las formaciones litológicas dentro de la geología y la tierra, algunas zonas potenciales de mineral hacen mejorar y entender el proceso que interviene en ellas en donde se incorporan más métodos en lo que se refiere a estudios de 
IIVESTIGACIÓn Y CIERCIA DE LA UNIVERSIDAD AUTÓNOMA DE RGUASCALIERTES focos de potencialidad magnética, así como la determinación de la cuantificación de los recursos naturales.

\section{Agradecimientos}

El autor desea agradecer los valiosos comentarios y contribuciones de Francisco Benita Maldonado y
Simón Eduardo Carranco Lozada, los cuales sirvieron para mejorar considerablemente la calidad de este documento. También los comentarios y sugerencias de dos dictaminadores anónimos, así como a Mónica Ávalos Valladares su sugerencia sobre el traslado de parte de los textos al pie de figuras a párrafo, que mejoró la estructura del trabajo.

\section{LITERATURA CITADA}

- AIKEN, C. L. V. et al. Integrated studies of Mexico with gravity, magnetic and GIS database. The Leading Edge, 16(12): 17791785, 1997.

- BHATTACHARYYA, B. K. Computer modeling in gravity and magnetic interpretation. Geophysics, 43(5): 912- 929, 1978.

- CENTENO GARCíA, E. et al. The Guerrero Composite Terrane of Western Mexico: Collision and subsequent rifting in a suprasubduction zone. The Geological Society of America, 436, 279308, 2008.

- $\quad$ CLARK, D. A. Magnetic petrophysics and magnetic petrology: aids to geologic interpretation of magnetic surveys. AGSO Journal of Australian Geology and Geophysics, 17(2): 83-103, 1997.

- DAMON, P. E. et al. Evolución de los arcos magmáticos en Mexico y su relación con la metalogénesis. Revista del Instituto de Geología de la Universidad Nacional Autónoma de Mexico, 5(2): 223-238, 1981.

- DAVIS, B. E. GIS: a visual approach. Albany, NY, US: Delmar Thomson Learning, 2001.

- FACCHINELLI, A. et al. Multivariate statistical and GISbased approach to identify heavy metal sources in soils. Environmental Pollution, 114 (3): 313-324, 2001.

- JAIN, A. K. y DUBES, R. C. Algorithms for Clustering Data. Englewood Cliffs, NJ, US: Prentice Hall, 1988.

- KEAREY, P. et al. An Introduction to Geophysical Exploration. Wiley-Blackwell Publishing, 2002.

- LÓPEZ LOERA, H. y TRISTÁN GONZÁlez, M. Geología y magnetometría aérea del Graben de Villa de Reyes, San Luis Potosí, Mesa Central de México: implicaciones tectónicas y geohidrológicas. Boletín de la Sociedad Geológica Mexicana, 65(1): 137-156, 2013.

- MILLIGAN, P. R. y GUNN, P. J. Enhancement and presentation of airborne geophysical data. AGSO Journal of Australian Geology and Geophysics, 17(2): 63-75, 1997.
- SGM (SERVICIO GEOLÓGICO MEXICANO). Carta GeologíaMinera, G14-C61, Escala 1:50000, 2004a.

- SGM (SERVICIO GEOLÓGICO MEXICANO). Carta GeologíaMinera, G14-C62, Escala 1:50000, 2004b.

- SGM (SERVICIO GEOLÓGICO MEXICANO). Carta GeologíaMinera, G14-C71, Escala 1:50000, 1996.

- SGM (SERVICIO GEOLÓGICO MEXICANO). Carta GeologíaMinera, G14-C72, Escala 1:50000, 2005.

- SGM (SERVICIO GEOLÓGICO MEXICANO). Carta magnética de la primera derivada vertical del campo total reducido al polo alta resolución G14-C61, Escala 1:50000, 1997.

- SGM (SERVICIO GEOLÓGICO MEXICANO). Carta magnética de la primera derivada vertical del campo total reducido al polo alta resolución G14-C671, Escala 1:50000, 2009.

- SGM (SERVICIO GEOLÓGICO MEXICANO). Carta magnética de la primera derivada vertical del campo total reducido al polo alta resolución G14-C672, Escala 1:50000, 2010.

- SGM (SERVICIO GEOLÓGICO MEXICANO). Carta magnética del campo total G14-C61, Escala 1:50000, 1997.

- SGM (SERVICIO GEOLÓGICO MEXICANO). Carta magnética del campo total G14-C62, Escala 1:50000, 2009.

- SGM (SERVICIO GEOLÓGICO MEXICANO). Carta magnética del campo total G14-C71, Escala 1:50000, 1997.

- SGM (SERVICIO GEOLÓGICO MEXICANO). Carta magnética del campo total G14-C72, Escala 1:50000, 1998.

\section{De páginas electrónicas}

- AMBRIZ AVENDAÑO, A. [Fotografía]. Recuperada el sábado 5 de marzo de 2016, de http://www.panoramio.com/ photo/ 18358508 